\title{
On the breakup of an air bubble injected into a fully developed turbulent flow. Part 1. Breakup frequency
}

\author{
By C. MARTÍNEZ-BAZÁN, \\ J. L. MONTAÑ́S AND J. C. LASHERAS \\ Department of Mechanical and Aerospace Engineering, University of Califormia, San Diego, \\ La Jolla, CA 92093-0411, USA
}

The transient evolution of the bubble-size probability density functions resulting from the breakup of an air bubble injected into a fully developed turbulent water flow has been measured experimentally using phase Doppler particle sizing (PDPA) and image processing techniques. These measurements were used to determine the breakup frequency of the bubbles as a function of their size and of the critical diameter $D_{c}$ defined as $D_{c}=1.26(\sigma / \rho)^{3 / 5} \epsilon^{-2 / 5}$, where $\epsilon$ is the rate of dissipation per unit mass and per unit time of the underlying turbulence. A phenomenological model is proposed showing the existence of two distinct bubble size regimes. For bubbles of sizes comparable to $D_{c}$, the breakup frequency is shown to increase as $(\sigma / \rho)^{-2 / 5} \epsilon^{3 / 5} \sqrt{D / D_{c}-1}$, while for large bubbles whose sizes are greater than $1.63 D_{c}$, it decreases with the bubble size as $\epsilon^{1 / 3} D^{-2 / 3}$. The model is shown to be in good agreement with measurements performed over a wide range of bubble sizes and turbulence intensities.

\section{Introduction}

The mass transfer rate occurring in many natural and engineering processes depends on the amount of contact surface which is created between two immiscible fluids. For example, in liquid-liquid or gas-liquid separators, the absorption rate of a certain chemical species depends on the distribution of droplet sizes in which one fluid is dispersed into another. In aeration processes such as the ones which occur naturally during the interaction of the atmosphere and the oceans, the absorption rate of carbon dioxide, or many other water-soluble trace species, depends on the amount of air entrained by the wave action, and more importantly on the distribution of bubble sizes resulting from the breakup of the entrained air by the underlying turbulence existing under the free surface, Melville (1996).

Central to the development of predictive models of all these engineering and natural processes is the description of the breakup of an immiscible fluid immersed in a turbulent one. Although this problem has been the subject of a continuing investigation beginning with the early work of Kolmogorov (1949), Baranaev, Tevenovskiy \& Tregubova (1949), and Hinze (1955), and has generated a large bibliography, a unified model capable of predicting the probability density function of the droplets (or bubbles) resulting from the turbulent breakup does not yet exist. Over the years, the chemical engineering community has devoted a considerable amount of work to 
the study of stirred (or agitated) vessels such as those used to produce emulsions, Coulaloglou \& Tavlarides (1977), Tsouris \& Tavlarides (1994), Prince \& Blanch (1990), Berkman \& Calabrese (1988), Konno, Aoki \& Saito (1983), among many others. Although these experiments have provided valuable information on the steady-state droplet size distribution, they have not been able to produce reliable information on the evolution of the droplet sizes during the transient breakup processes, knowledge which is essential to the development of the models. Furthermore, due to the complexity introduced by the use of turbine impellers in the agitated tank experiments, the nature of the turbulence existing in the tank is difficult to characterize (Cutter 1996; Calabrese, Chang \& Dang 1986a; Calabrese, Wang \& Bryner 1986b; and others), and often precludes the interpretation of these measurements.

Essential to the development of the models is knowledge of both the probability (or frequency) of the drop's (or bubble) breakup, and of the resulting probability density distribution of the size of the daughter droplets. In the past, there have been numerous experimental studies to determine the drop's breakup frequency. For example, many attempts have been made to determine this frequency in stirred tanks, or in turbulent pipe flows, by measuring the time evolution of the drop-size p.d.f.s (Sathyagal, Ramkrishna \& Narsimhan 1994; Sathyagal \& Ramkrishna 1996; Narsimhan, Gupta \& Ramkrishna 1979; Narsimhan, Nejfelt \& Ramkrishna 1984; Nambiar et al. 1992, 1994; and others). As mentioned above, the difficulty in the use of stirred vessels is that the turbulence in the vessel is not very well characterized since it is not only inhomogeneous throughout the vessel, but more importantly, it is highly anisotropic consisting of high-shear regions on the surface of the impeller and strong tip vortices shed by the impeller blades. Furthermore, in these vessel experiments, the transient size p.d.f.s are often measured by withdrawing samples over time which are later diluted, or stabilized, prior to their measurements. These sampling techniques neither guarantee that the droplet-size p.d.f.s are frozen, nor that they are preserved during the sampling. On the other hand, the drop or bubble breakup experiments conducted in turbulent pipe flows have had similar difficulties also resulting from the anisotropic nature of the turbulence in the pipe which is composed of free-stream turbulence and high-shear regions near the walls. These shear regions often cause the preferential migration of the bubbles towards the wall making the characterization of the turbulence under which the breakup takes place very difficult.

With these difficulties in mind, the objective of the present study is, therefore, to conduct a systematic set of measurements of the bubble breakup frequency under well-controlled, and well-characterized turbulent conditions. In order to isolate the problem, and to prevent the additional complexity introduced by the use of turbines or any other moving surfaces to generate the turbulence, we selected to study the turbulent breakup by injecting air bubbles into the fully developed turbulent region along the central axis of a high Reynolds number water jet. Through the use of phase Doppler techniques (PDPA) and image processing. we then measured the transient p.d.f.s of the bubbles sizes resulting from the turbulent breakup over a wide range of initial bubble sizes and turbulent conditions characterized by the turbulent kinetic energy (or the dissipation rate) of the underlying turbulence. This approach allowed us to minimize the undesirable effects that have plagued previous experiments, and to systematically study the turbulent breakup frequency using non-intrusive optical techniques under well-defined, nearly homogeneous turbulence conditions. Furthermore, it also provided for isolating the turbulent breakup process from the effects of buoyancy.

The organization of the paper is as follows. In $\$ 2$, we discuss the experimental 

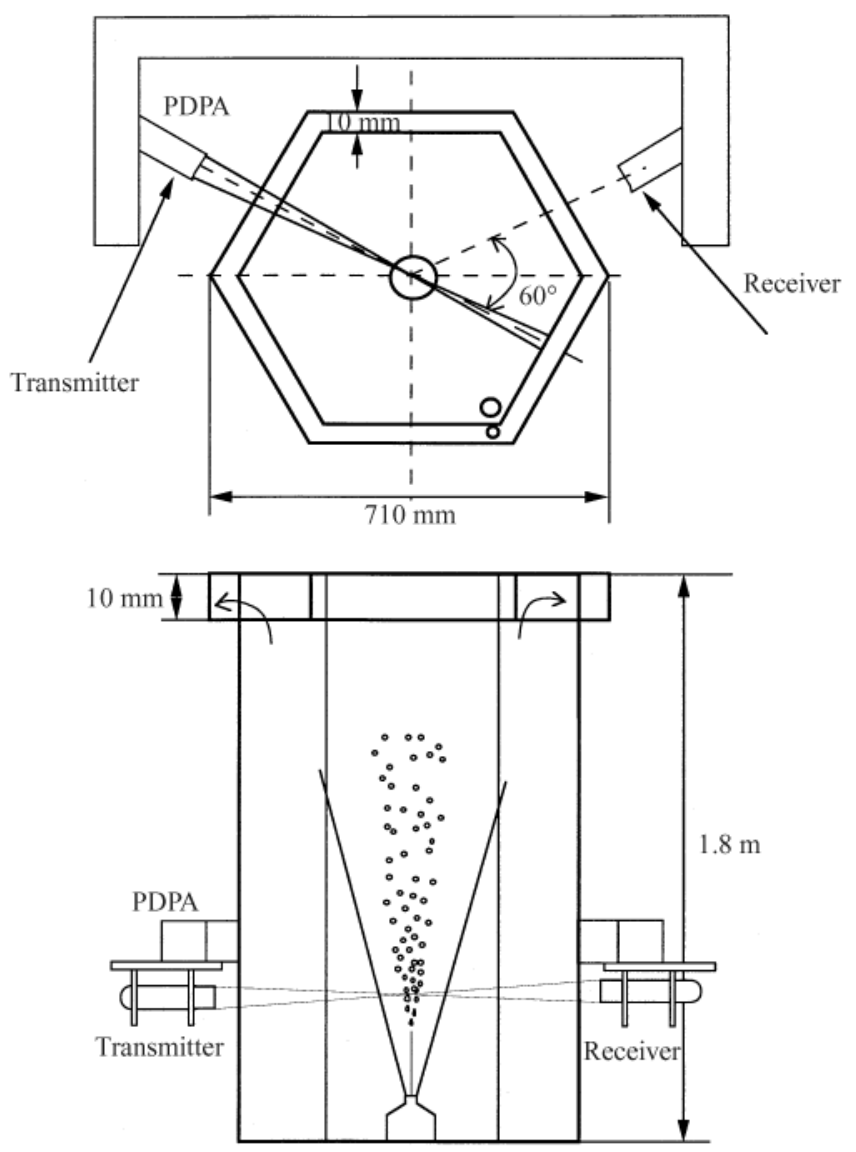

FIGURE 1. Experimental facility.

method and measuring techniques used. In $\S 3.1$, we present a detailed set of measurements of the transient bubble-size p.d.f. obtained over a wide range of bubble sizes and turbulence intensities. The measured breakup frequency as a function of both the bubble size and the local value of the turbulence intensity is discussed in $\S 3.2$. Finally, a phenomenological model is proposed in $\S 4$, and compared with the experimental results.

\section{Experimental setup}

The experimental facility, shown in figure 1, consisted of a submerged water jet where air was injected through a small hypodermic needle at a given position along its centreline. In order to maximize the accuracy of the phase Doppler and other optical measurements, the tank in which the jet discharges was designed with a hexagonal cross-section. The water jet nozzle was located at the bottom and the jet discharges vertically upwards into the tank. In order to minimize the recirculating flow produced in the tank by the high-momentum water jet, the water was allowed to overflow from the top of the tank through a set of gutters placed on each side. Uniform velocity was achieved at the exit of the nozzle of the submerged water jet by using two perforated plates located upstream of a high-contraction-ratio $(250: 1)$ nozzle, as seen in figure 2. Although different nozzle exit diameters can be used, in all 


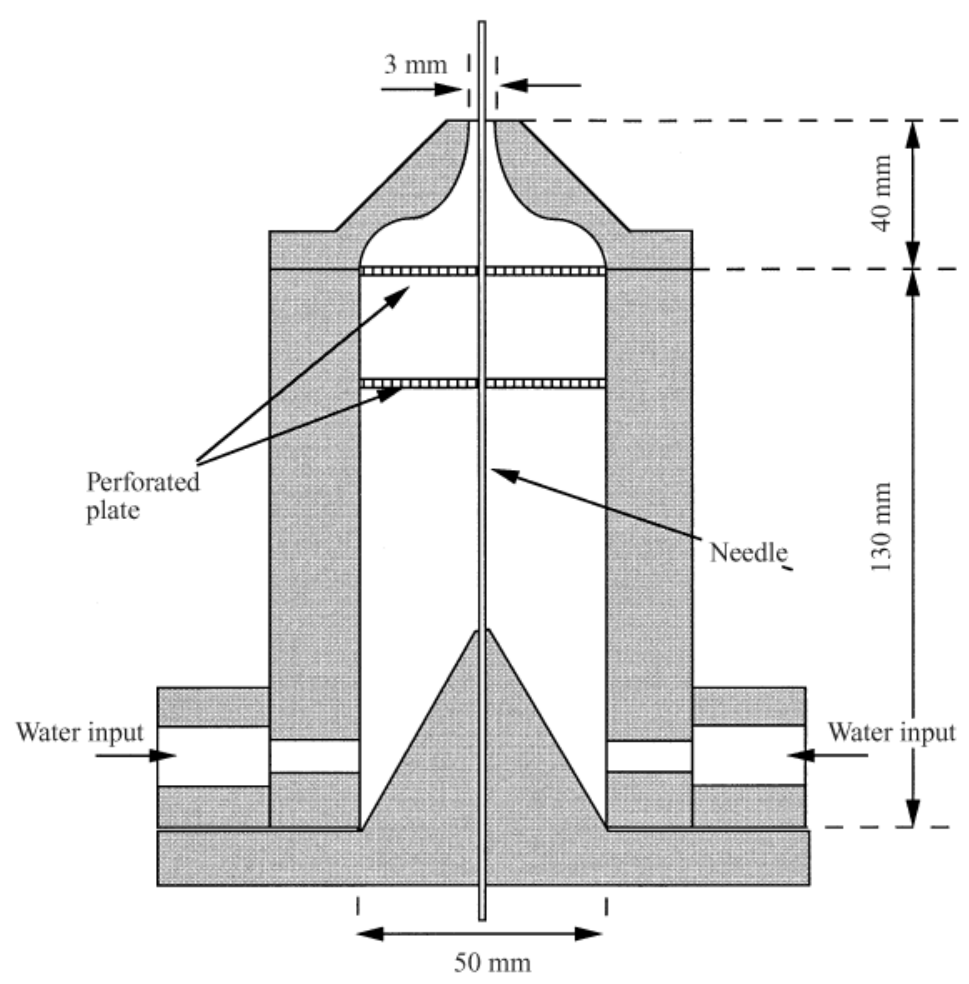

Figure 2. Detail of the water jet nozzle and air injection needle.

the experiments reported here it was $3 \mathrm{~mm}$. The jet Reynolds number, $R_{e}=U_{0} D_{J} / v$, calculated with the velocity at the exit of the water jet, $U_{0}$, the diameter of the nozzle, $D_{J}$, and the kinematic viscosity of the water, $v$, can be systematically varied in our experiments from $2.5 \times 10^{4}$ to $9 \times 10^{4}$. Air was injected coaxially at a selected downstream location on the axis of the submerged turbulent water jet through a small hypodermic needle whose cross-sectional area was always less than 60 times the cross-sectional area of the exit nozzle of the water jet. To avoid any undesirable vibration effects at the air injection point, the needle was supported at the crossing points with the two perforated plates, as shown in figure 2. The bubble injection point, which determines the value of the turbulent kinetic energy of the underlying turbulence where the bubble breakup process takes place, can be varied along the axis of the water jet from 10 to 50 jet diameters by moving the needle vertically. All the experiments reported here, however, correspond to injection points located between 15 and 25 jet diameters downstream from the nozzle exit section. These positions are several diameters downstream from the end of the potential cone region of the water jet, and our measurements of the energy spectrum indicated that the turbulence is fully developed in the scales of interest to our problem in all Reynolds number cases studied.

The water flow rate, $Q_{w}$, can be varied from $5.83 \times 10^{-5} \mathrm{~m}^{3} \mathrm{~s}^{-1}$ to $2.1 \times 10^{-4} \mathrm{~m}^{3} \mathrm{~s}^{-1}$ providing, for the $3 \mathrm{~mm}$ diameter nozzle, a range of exit velocities, $U_{0}$, from $8.25 \mathrm{~m} \mathrm{~s}^{-1}$ to $30 \mathrm{~m} \mathrm{~s}^{-1}$. The flow rate of air, $Q_{a}$, can be increased from $5.8 \times 10^{-8} \mathrm{~m}^{3} \mathrm{~s}^{-1}$ to $1.25 \times 10^{-6} \mathrm{~m}^{3} \mathrm{~s}^{-1}$. From the one-dimensional spectrum of the fluctuating component of the axial velocity, and considering the turbulence to be locally homogeneous and isotropic, the integral scale, $L_{x}$, the dissipation rate of turbulent kinetic energy per 


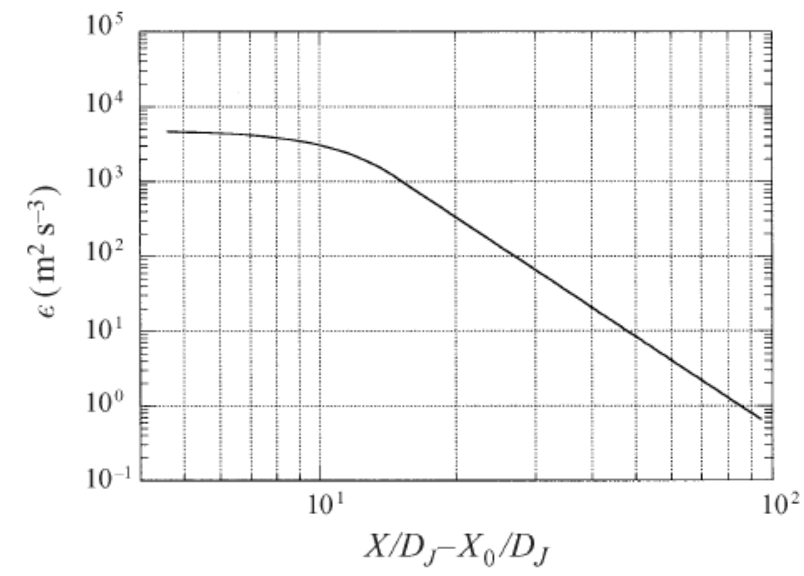

FIGURE 3. Downstream evolution of the dissipation rate of TKE, $\epsilon . X_{0} \approx 5.4 D_{J}$ indicates the virtual origin, the velocity at the exit of the nozzle is $U_{0}=17 \mathrm{~m} \mathrm{~s}^{-1}$.

unit mass and per unit time, $\epsilon$, the Taylor microscale, $\lambda_{t}$, and the Reynolds number based in the Taylor scale, $R_{\lambda_{t}}$, can be estimated as:

$$
\left.\begin{array}{l}
L_{x}=\frac{\pi E_{11}\left(k_{1}=0\right)}{2 \overline{u^{\prime 2}}}, \quad \epsilon=15 v \int_{0}^{\infty} k_{1}^{2} E_{11}\left(k_{1}\right) \mathrm{d} k_{1}, \\
\frac{1}{\lambda_{t}^{2}}=\frac{\epsilon \overline{u^{\prime 2}} v}{30}, \quad R_{\lambda_{t}}=\frac{u^{\prime} \lambda_{t}}{v},
\end{array}\right\}
$$

where $u^{\prime}$ is the fluctuating axial velocity, see Hinze (1959), Gibson (1963), Friehe, Van Atta \& Gibson (1972). We conducted an extensive set of measurements using both hot-film anemometry and LDV to characterize the turbulence of the submerged water jet used in all our experiments. In all cases, our measurements showed that our jet behaved as a well-known high Reynolds number, turbulent, free jet, see Gibson (1963), Antonia, Satyaprakash \& Hussain (1980), Hussein, Capp \& George (1994). Furthermore, by conducting the water velocity measurements with and without air injection, we were also able to confirm that the evolution of our free jet was totally unaffected by the presence of the very small void fraction occupied by the bubbles.

The behaviour of the water jet as a free jet is a result of the following design features:

(a) our small diameter water jet discharged into a very large water tank whose cross-sectional area is 50000 times larger than that of the jets nozzle;

(b) the height of the tank was 600 times the jet diameter;

(c) the momentum carried out by the water jet upon reaching the free surface of the tank was removed from the system by a carefully designed overflow drainage;

(d) the running time of all our measurements from the starting time of the water jet, to achievement of the steady-state condition, to injection of the air jet, and to completion of the bubble size measurements always took fewer than $10 \mathrm{~s}$. Thus, no measurable recirculation flow was created in the tank by entrainment flow of the submerged jet.

Consequently, since the turbulent characteristic of a high Reynolds number, free, axisymmetric jet are well known, there is no need to provide further details.

In order to measure the breakup frequency of the bubbles as a function of their size and of the turbulent kinetic energy (TKE) of the underlying turbulence, we conducted 
a number of experiments in which we varied systematically both the initial bubble size and the value of the TKE of the underlying turbulence at the location of the air injection point. By varying the location of the injection point, the Reynolds number of the water jet, and the diameter of the air injection needle, we were able to vary not only the dissipation rate of the turbulent kinetic energy of the surrounding water, but also the initial size p.d.f. of the bubbles. In our experiments, we measured this initial bubble size distribution within the first measuring window and subsequently followed its evolution. With our experimental set-up, while we may not have had complete a priori control of the initial bubble size p.d.f., we could produce different initial bubble p.d.f.s and then could follow their subsequent breakup as they were convected into regions of known turbulence characteristics.

All experiments were performed in the following way. The desired initial value of the turbulence kinetic energy was selected by positioning the air injection needle at a given downstream distance from the nozzle of the water jet. In all cases, the selected downstream distances were greater than or equal to $15 \mathrm{D}$, to ensure a region of fully developed turbulence. After injecting the air bubbles, we followed the evolution of their breakup as they were convected downstream by the mean axial velocity of the jet to regions of lower and lower turbulent kinetic energy. During this evolution, buoyancy effects can be shown to be negligible since the terminal velocities of all the bubbles were always an order of magnitude smaller than the mean velocity of the jet, and the time needed for the bubbles to reach their terminal velocity was one order of magnitude larger than the residence time in the region of interest. Since in the water jet the turbulence kinetic energy (or the dissipation rate, $\epsilon$ ) decays with the downstream distance as shown in figure 3 (our measurements, performed for all flow conditions, agreed with those reported in the literature, i.e. Antonia et al. 1980; Friehe et al. 1972), we performed measurements of the transient bubble-size p.d.f.s in 15 discrete downstream regions (or windows) as indicated in figure 4 . Notice that, since we knew both the residence time and the value of the underlying turbulent kinetic energy in each window, we therefore could measure all the parameters needed in each experiment in order to be able to calculate the bubble breakup frequency as a function of the bubble's size and $\epsilon$. The length of each window along the axial direction was chosen such that $\epsilon$ varied a maximum of $10 \%$ along its length and, thus, it could be assumed to be nearly constant. Furthermore, the cross-stream width of the window was always selected to be large enough to include all the region where the bubbles resulting from the breakup were dispersed by the turbulence. A summary of the various flow conditions selected for our measurements is given in table 1 . Note that in our experiments, in addition to varying $\epsilon$ by changing the location of the injection point, we also varied both the diameter of the injection needle, $D_{a}$, and the air injection velocity, $U_{a}$, resulting in a variation of the initial bubble diameter $D_{0}$.

Since bubble sphericity is a necessary condition for the use of phase Doppler techniques, the evolution of the bubble-size probability density function in the region of interest in our experiments, where bubbles are not spherical, was always measured by means of digital image analysis. Only when the breakup was finished, and the bubbles were nearly spherical, with their turbulent Weber number less than unity, was phase Doppler anemometry (PDA) used to measure the bubble-size p.d.f.s.

The images were taken by illuminating the flow with uniform, diffused white light and capturing the image with a Sony XC-77R CCD camera placed in front of the light source using a short exposure time of $1 / 80000 \mathrm{~s}$. The $768(\mathrm{H}) \times 493(\mathrm{~V})$ images were captured with a $640 \times 480$ pixel resolution frame grabber and stored in a computer for later processing. To maximize the resolution of our measurements, the camera 


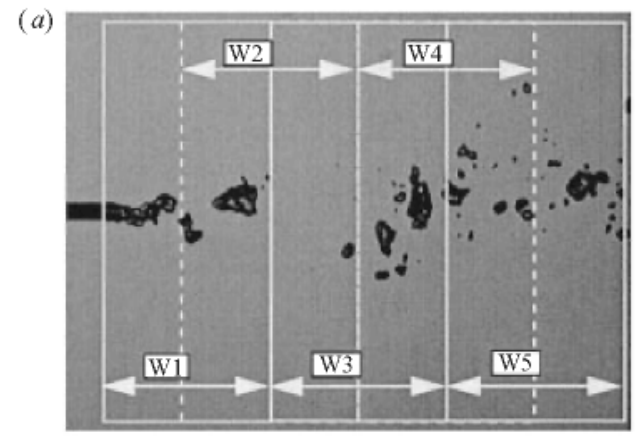

(b)

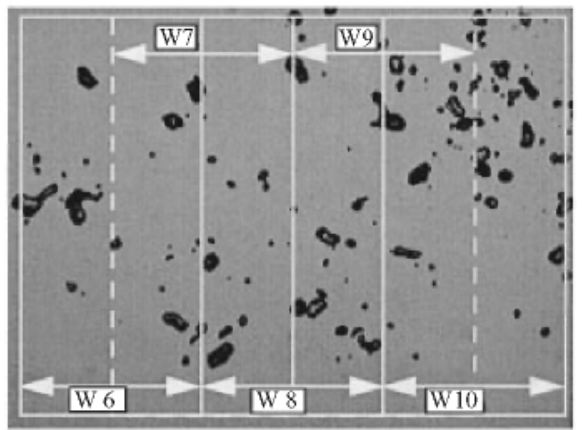

(c)

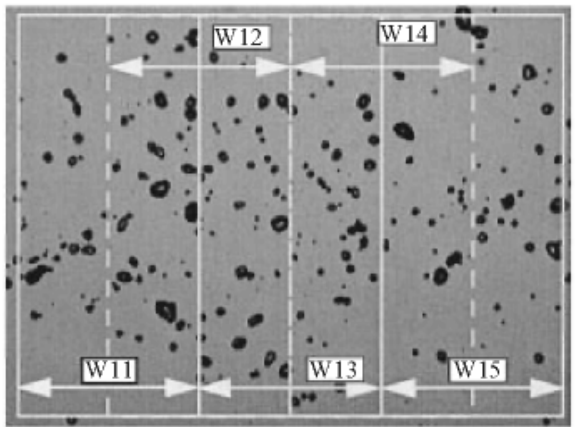

FIGURE 4. Discretization of the flow region where the bubble breakup takes place. (a) Section 1, windows 1-5. (b) Section 2, windows 6-10. (c) Section 3, windows 11-15. The downstream length of each measuring window indicated by arrows is $7.14 \mathrm{~mm}$. Experimental Set 3a. Flow goes from left to right in each picture.

was focused on a small region of $2.3 \mathrm{~cm} \times 1.7 \mathrm{~cm}$, and to resolve the entire breakup region while maintaining the desired resolution, the camera was traversed vertically to three positions $2.3 \mathrm{~cm}$ apart. Each image was then divided into five windows of equal size as indicated in figure 4 . Each window consisted of a $7.14 \mathrm{~mm} \times 16.25 \mathrm{~mm}$ rectangle of the flow and was digitized with a resolution of $200 \times 455$ pixels. This digitization allowed a pixel-size resolution of $30 \mu \mathrm{m}$, which resulted in a minimum measurable bubble diameter of $83 \mu \mathrm{m}$ composed by approximately 6 pixels. Although the resolution obtained for the smallest bubble size is not very high, it does not affect the results presented in this paper. As it will be shown later, the bubbles used in this work to measure the breakup frequency were always larger than $1 \mathrm{~mm}$, and therefore, their images consisted always of more than 870 pixels. To increase the 


$\begin{array}{rccccc} & \begin{array}{c}D_{a} \\ (\mathrm{~mm})\end{array} & \begin{array}{c}U_{0} \\ \left(\mathrm{~m} \mathrm{~s}^{-1}\right)\end{array} & R_{e}=\frac{U_{0} D_{j}}{v} & \begin{array}{c}U_{a} \\ \left(\mathrm{~m} \mathrm{~s}^{-1}\right)\end{array} & \begin{array}{c}\text { Injection point } \\ X / D_{j}\end{array} \\ \text { Set 1 } & 0.394 & 17.0 & 51000 & 8.88 & 25 \\ \text { Set 2 } & 0.394 & 17.0 & 51000 & 9.84 & 15 \\ \text { Set 3a } & 0.394 & 17.0 & 25500 & 9.84 & 15 \\ \text { b } & 0.584 & 17.0 & 25500 & 4.48 & 15 \\ \text { c } & 1.194 & 17.0 & 25500 & 1.07 & 15\end{array}$

TABLE 1. Experimental conditions. $R_{e}$ has been calculated based on the exit velocity, $U_{0}$, and the open section at the exit of the nozzle, $D_{j}$. This open section was kept constant in the three cases of Set 3 .

spatial resolution of the discretization, the measuring windows were overlapped $50 \%$ of their length as indicated in figure 4.

At all vertical positions of the CCD camera, we recorded 1000 frames. Each frame was first subtracted from the background illumination and subsequently an edge detection threshold operation was applied. From this, binary images as shown in figure 5 were produced. From the binary images, we computed the projected area of the bubbles. Since the underlying turbulence is isotropic, the mean shape of the projection of the bubble on any given plane is assumed to be statistically the same and independent of its orientation, thus allowing the calculation of an equivalent bubble diameter. Using this method, a bubble-size histogram was measured from each of the 15 windows. From each histogram, the bubble-volume probability density function, V.p.d.f., was then calculated. The above described digital image processing method was first calibrated against well-known bubble geometries in the range of bubble diameters produced in our experiments and found to be accurate within $10 \%$, see Martínez-Bazán (1998). For the very low air void fraction generated in our experiments, and the small depth of the field used to acquire our images, we are confident that the equivalent size measured is within the above estimated accuracy.

Before beginning the discussion of the measurements, it is important to emphasize that in all our experiments the bubbles broke up under the action of fully developed, isotropic turbulence, which was spatially nearly uniform. The air was always injected at the jet's centre axis, and during their breakup, the bubbles remained at the centre of the jet, being transported laterally by the action of the turbulence to radial distances always smaller than $30 \%$ of the width of the jet. A schematic of the flow conditions under which all the breakup experiments were conducted, showing all the important dimensions of interest in our experiments, is given in figure 6 . In all cases, the characteristic width of the jet in the region of interest, $D_{w_{j}}$, was always three to five times larger than the lateral dimension of the measuring window, $l_{1}$, which in turn was always larger than the maximum radial distance where the bubbles were dispersed by the turbulence. Within this central region of the jet, the turbulent kinetic energy was measured to be nearly uniform, a result consistent with early measurements by Hussein et al. (1994). In agreement with measurements of high Reynolds number axisymmetric jets, i.e. Wygnanski \& Fiedler (1969). Hussein et al. (1994), the measured r.m.s. of the axial and transversal components of the jet velocity, $u^{\prime}, v^{\prime}$, were always nearly uniform throughout the volume of interest within $3 \%$ to $5 \%$.

Furthermore, in all our experiments, owing to the very short residence time of the 
(a)

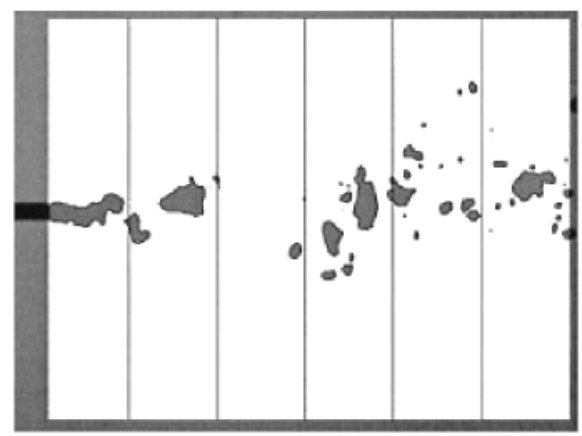

(b)

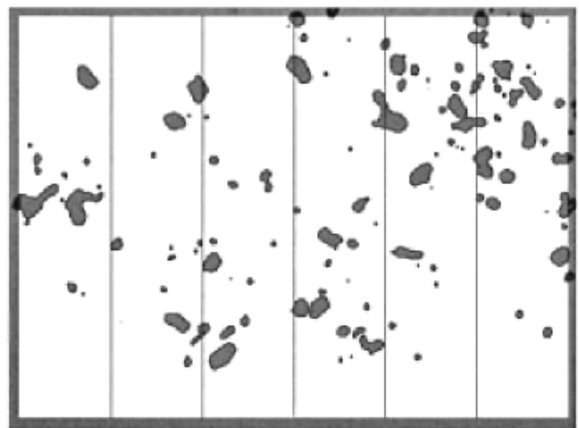

(c)

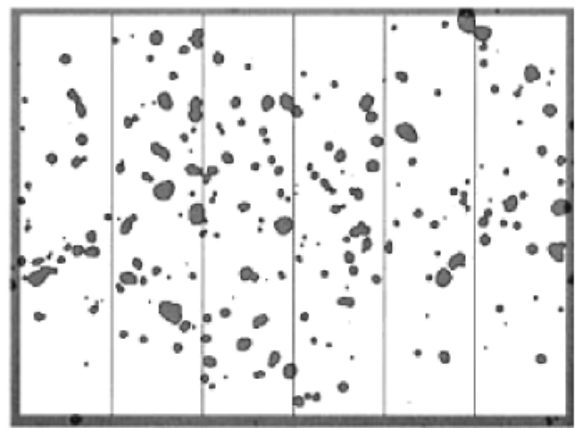

FIGURE 5. Binary images corresponding to the flow conditions shown in figure 4. (a) Section 1. (b) Section 2. (c) Section 3.

bubbles in the breakup region, neither buoyancy effects nor the dynamics of bubble oscillations play any role in the bubble breakup.

\section{Experimental results}

\subsection{Evolution of the bubble-volume p.d.f.s}

Let us begin considering the cases of air injection at $X / D_{J}=15$ and $U_{0}=17 \mathrm{~m} \mathrm{~s}^{-1}$, which correspond to a Reynolds number of the water jet $R e=25500$ (Set 3 in table 1). Under these conditions, we investigated three different air injection diameters, $D_{a_{1}}=0.394 \mathrm{~mm}, D_{a_{2}}=0.584 \mathrm{~mm}, D_{a_{3}}=1.194 \mathrm{~mm}$. In each of the three diameter cases, the injection velocity of the air was adjusted to give always the same flow 


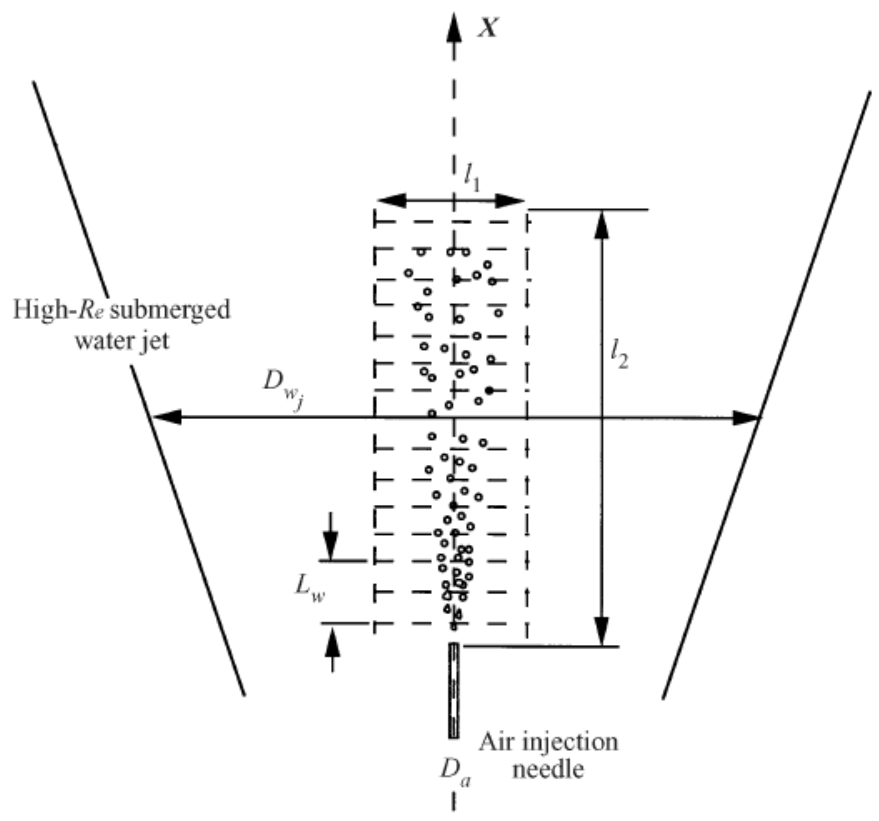

FiguRE 6. Schematic representation of conditions used for the turbulent breakup measurements. Characteristic water jet diameter, $D_{w_{j}}$, the width and length of all measuring windows was respectively $l_{1}=16.25 \mathrm{~mm}$ and $L_{w}=7.14 \mathrm{~mm}$. In all experiments $l_{1} / D_{w_{j}}<0.3$.

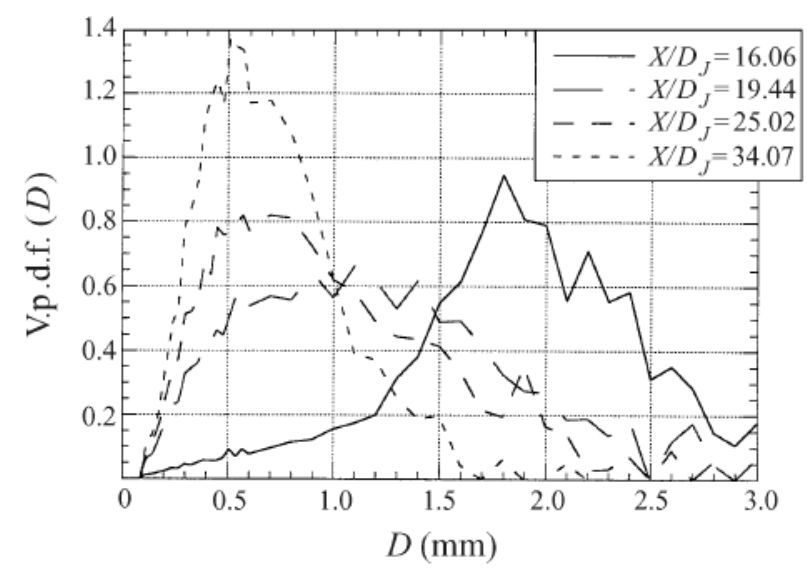

FIgURE 7. Evolution of the bubble V.p.d.f., $D_{a}=1.194 \mathrm{~mm}$. Experimental Set 3c.

rate, $Q_{a}=72 \mathrm{ml} \mathrm{min}^{-1}$. For the case of the largest needle diameter, the air injection velocity was selected to be equal to the mean velocity of the water jet at the point of injection. Thus, when the air exits the needle it is only exposed to the turbulent stresses resulting from the fluctuating component of the water velocity. In other words, the bubbles only see the nearly homogeneous, isotropic turbulent flow existing on the jet's centreline at the injection point. The evolution of the bubble-volume p.d.f. resulting from the turbulent breakup of the air as it is convected to regions of decaying dissipation rate, $\epsilon$, is shown in figure 7. For clarity we have only plotted four measuring locations (windows). 
When the air is injected into the water, the velocity fluctuations of the underlying turbulence of the jet results in deformation forces that are much greater than the confinement forces due to surface tension and the bubble breaks. However, the probability of breakup depends on the characteristic size of the bubble, $D$, and on the value of the turbulent kinetic energy (or dissipation rate, $\epsilon$ ) of the underlying turbulence. For each value of $\epsilon$, a critical capillary length, $D_{c}$, exists such that, in the mean, the turbulent stresses are equal to the surface tension forces. This critical capillary diameter, $D_{c}$ is given by $D_{c}=1.26(\sigma / \rho)^{3 / 5} \epsilon^{-2 / 5}$. In this particular experiment the value of the critical capillary length was $D_{c} \approx 264 \mu \mathrm{m}$ at the point of injection of air. Note that, since initially $D \gg D_{c}$, the bubble begins to break at a certain frequency right at the injection point, and at the first measuring location. $X / D_{J}=16.06$, the volume p.d.f. already shows a large peak at $D \approx 1.7 \mathrm{~mm}$ with relatively wide tails. The shape of the V.p.d.f. appears to be almost a symmetric distribution confined between $0.5 \mathrm{~mm}$ and $2.5 \mathrm{~mm}$. As the bubbles are transported downstream by the mean motion of the water jet to the next measuring location. they continue breaking at a rate determined by the local value of the intensity of the turbulent kinetic energy (or dissipation rate). Thus, at $X / D_{j}=19.44$ (window number 4), the measured bubble-volume p.d.f. is observed to have evolved considerably to a new shape which has resulted from the rapid decay in the number of large sized bubbles and the associated increase in the number of smaller ones. This breakup process continues while the bubbles are convected downstream to regions of lower and lower dissipation rate, $\epsilon$, until eventually we observed that the V.p.d.f. reaches a frozen or unchanged shape, shown in figure $8(a)$. The existence of a frozen state is expected, since, as the bubbles are broken by the turbulence stresses from the liquid, their size monotonically decreases with the downstream distance. In each case the rate of decay of the bubbles' size is obviously associated with the local value of $\epsilon$ at the injection point and the convective velocity at which they are transported to regions of decaying $\epsilon$. As $\epsilon$ decays with the downstream distance $\left(\epsilon \alpha_{\epsilon}\left(X / D_{j}\right)^{-4}\right)$, see figure 3 , the critical diameter, $D_{c}$, also increases as $\left(X / D_{J}\right)^{8 / 5}$. As a result of these combined effects, a downstream distance exists where all the bubbles become smaller than $D_{c}$, at which point no further breakup takes place, and the p.d.f. will remain frozen from that point on. In the above described experiment, this frozen p.d.f. is achieved at $X / D_{j} \approx 26$. Note in figure $8(a)$ that the bubble-volume p.d.f.s measured at the last three measuring stations are almost identical.

A statistical description of the bubble distribution can be obtained by using the distribution function $p(D, x, v, t) \mathrm{d} D \mathrm{~d} x \mathrm{~d} v$ which is defined as the probable number of bubbles with diameters in the range $\mathrm{d} D$ about $D$, located in the spatial range $\mathrm{d} x$ about the position $x$, with a velocity range $\mathrm{d} v$ about $v$, at time $t$. A Boltzmann-type equation often referred to as the population balance equation can be written to describe the time rate of change of the distribution function $p$,

$$
\frac{\partial p}{\partial t}+\nabla_{x} \cdot(v p)+\nabla_{v} \cdot(\boldsymbol{F} p)=-\frac{\partial}{\partial D}(R p)+\dot{Q}_{b}^{\prime}+\dot{Q}_{c}^{\prime}+\Gamma,
$$

where the rate of change of $p$ with time due to the bubble breakup and coalescence are denoted by $\dot{Q}_{b}^{\prime}$ and $\dot{Q}_{c}^{\prime}$ respectively. The force per unit mass acting on a bubble is denoted by $\boldsymbol{F}$, and the rate of change with time of the diameter of the particle due to evaporation, condensation, or dissolution is given by $R$. $\Gamma$ represents the rate of change of the distribution function caused by collisions which do not result in coalescence. In the case of interest here of very diluted systems, and immiscible fluid, coalescence and changes in $p$ due to bubble/bubble collisions can be neglected on the 

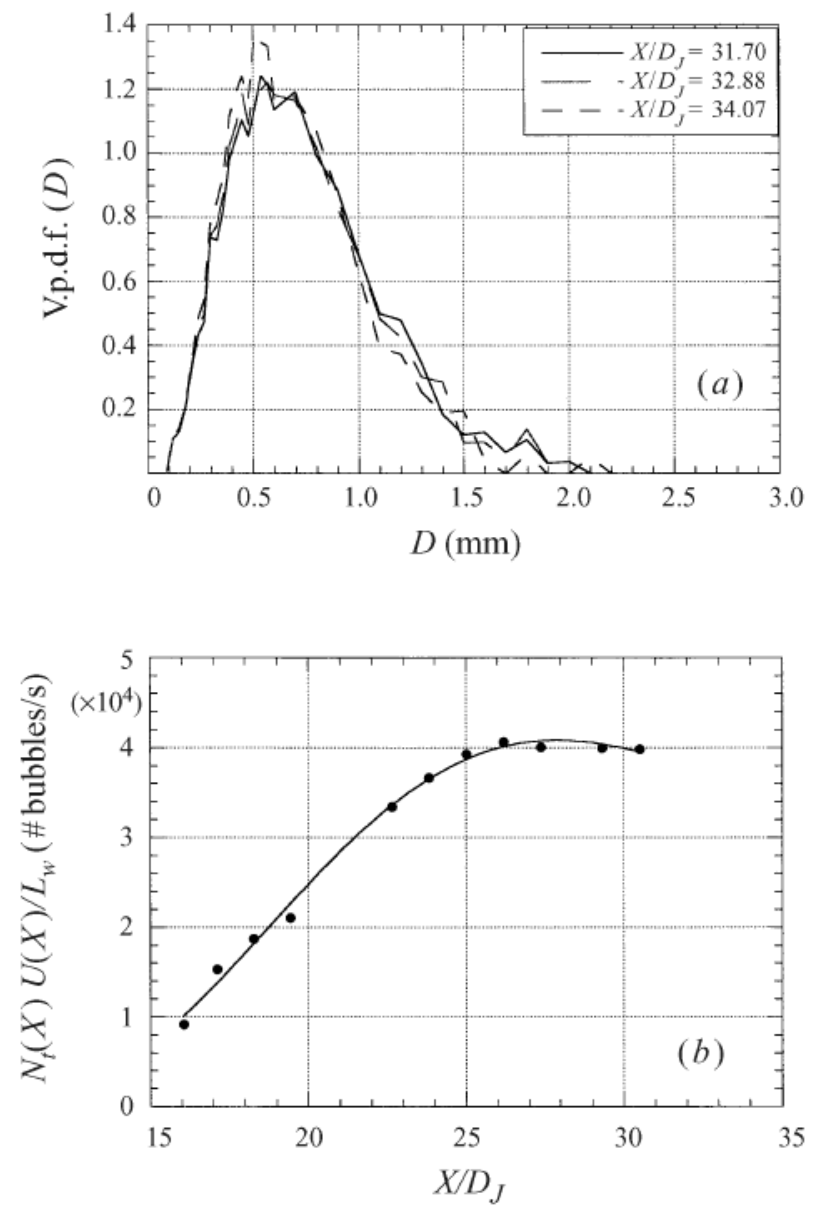

FiguRE 8. (a) Evidence of the existence of a frozen V.p.d.f., which remains unchanged in the last three measuring windows. (b) Evolution of the flux of bubbles per window. The value refers to the total flux, $N_{t} U / L_{w}$, obtained in each measuring window. Observe that after a frozen V.p.d.f. is achieved, the flux remains unchanged, $\approx 4 \times 10^{4}$ bubbles/s, indicating the termination of the breakup process. $L_{w}$ is the length of the measuring window. $D_{a}=1.194 \mathrm{~mm}$, experimental Set $3 \mathrm{c}$.

time scales studied, and the above equation reduces to

$$
\frac{\partial p}{\partial t}+\nabla_{x} \cdot(\boldsymbol{v} p)+\nabla_{v} \cdot(\boldsymbol{F} p)=\dot{Q}_{b}^{\prime},
$$

Integrating over the whole velocity space one obtains

$$
\frac{\partial n}{\partial t}+\nabla_{x} \cdot(\overline{\boldsymbol{v}} n)=\int_{D}^{\infty} m\left(D_{0}\right) f\left(D, D_{0}\right) g\left(D_{0}\right) n\left(D_{0}\right) \mathrm{d} D_{0}-g(D) n,
$$

where $n(D, \boldsymbol{x}, t)=\int p \mathrm{~d} \boldsymbol{v}$ is the number of bubbles per unit volume with size $D$ at a location $\boldsymbol{x}$ and time $t$, and $\overline{\boldsymbol{v}}$ is the mean velocity of all bubbles of size $D$ at a location $\boldsymbol{x}$ and time $t . g(D)$ is the bubble breakup frequency, $m\left(D_{0}\right)$ is the mean number of bubbles resulting from the breakup of a mother bubble of size $D_{0}$, and $f\left(D, D_{0}\right)$ is the size distribution of daughter bubbles formed from the breakage of a mother bubble of size $D_{0}$. 
In our steady-state, quasi-one-dimensional experiments, equation (3.3) reduces to

$$
\frac{\partial(\bar{v} n)}{\partial x}=\int_{D}^{\infty} m\left(D_{0}\right) f\left(D, D_{0}\right) g\left(D_{0}\right) n\left(D_{0}\right) \mathrm{d} D_{0}-g(D) n,
$$

where $x$ is the coordinate along the axis of the jet and $\bar{v}$ is the streamwise velocity of bubbles of size $D$. Furthermore, our PDPA measurements indicated that $\bar{v}$ was always approximately equal to the local mean velocity of the liquid $U(x)$.

One can use equation (3.4) to calculate the rate of change of the total number of bubbles of all sizes per unit volume, $n_{t}=N_{t} / A L_{w}$.

$$
\frac{\partial\left(n_{f} U\right)}{\partial x}=B_{i}-D_{e}
$$

where $N_{t}$ is the total number of bubbles measured in the entire volume of the measuring window which has a length $L_{w}$ and cross-sectional area $A, U$ is the convective velocity (which in our experiments was measured to be the same for all the bubbles regardless of their size and equal to the local velocity of the water jet), and $B_{i}=\iint_{D}^{\infty} m\left(D_{0}\right) f\left(D, D_{0}\right) g\left(D_{0}\right) n\left(D_{0}\right) \mathrm{d} D_{0} \mathrm{~d} D$ and $D_{e}=\int g(D) n \mathrm{~d} D$ stand for the rate of birth and death of the bubbles respectively due to breakup. In our experiments the residence time of the bubbles in the measuring region is so small that dissolution effects can be shown to be negligible. Furthermore, the bubble void fraction is always less than $10^{-5}$, and coalescence effects can also be neglected. A detailed discussion of the effect of the bubble coalescence and the lack of relevance to our experiments can be found in Martínez-Bazán (1998).

When the bubble breakup process is finished, $B_{i}=D_{e}=0$. Since we are looking at the steady-state problem $\left(\partial N_{t} / \partial t=0\right)$, the end of the breakup is marked by the downstream location where $\partial\left[N_{t}(x) U(x)\right] / \partial x=0$, or equivalently, the location where $N_{i}(x) U(x)$ becomes constant. Figure $8(b)$ shows the downstream evolution of the total flux of bubbles of all sizes, $N_{t}(x) U(x) / L_{w}$, indicating the existence of a frozen state. Observe that, near the injection point, while the bubbles reside in regions of high dissipation rate of turbulent kinetic energy, $N_{i}(x) U(x) / L_{w}$ sharply increases until reaching, further downstream, an asymptotic value of about $4 \times 10^{4}$ bubbles/s, at which point no more breakup occurs. It is important to recall that the width of our measuring window spans the entire small radial region where the bubbles are dispersed by the turbulence. Thus, dispersion effects are not influencing our measurements, and, indeed, we are measuring all the bubbles resulting from the breakup at each downstream location.

The measurements corresponding to the same flow conditions but with a needle injection diameter of $D_{a_{1}}=0.394 \mathrm{~mm}$ and $D_{a_{2}}=0.584 \mathrm{~mm}$ are shown in figures 9 and 10 respectively. To maintain the injection rate of air equal to the previous case while varying the needle's diameter, the air injection velocity was increased to $4.48 \mathrm{~m} \mathrm{~s}^{-1}$ and $9.84 \mathrm{~ms}^{-1}$ respectively. In these cases, the air exiting the needle is quickly decelerated to the mean velocity of the water jet in approximately $1 \mathrm{~mm}$, a length which is always an order of magnitude smaller than the dimension of the first measurement window. Thus, as was the case with the largest diameter needle, the bubbles breakup only under the effect of the underlying fully developed turbulence existing on the axis of the water jet.

Qualitatively, the measured evolution of the bubble-volume p.d.f. in these two cases appears to be similar to that described for the largest injection needle. However, it should be pointed out that the initial bubble p.d.f. (measured at the first window) is narrower as the needle diameter is decreased. This also results in a decrease in the 

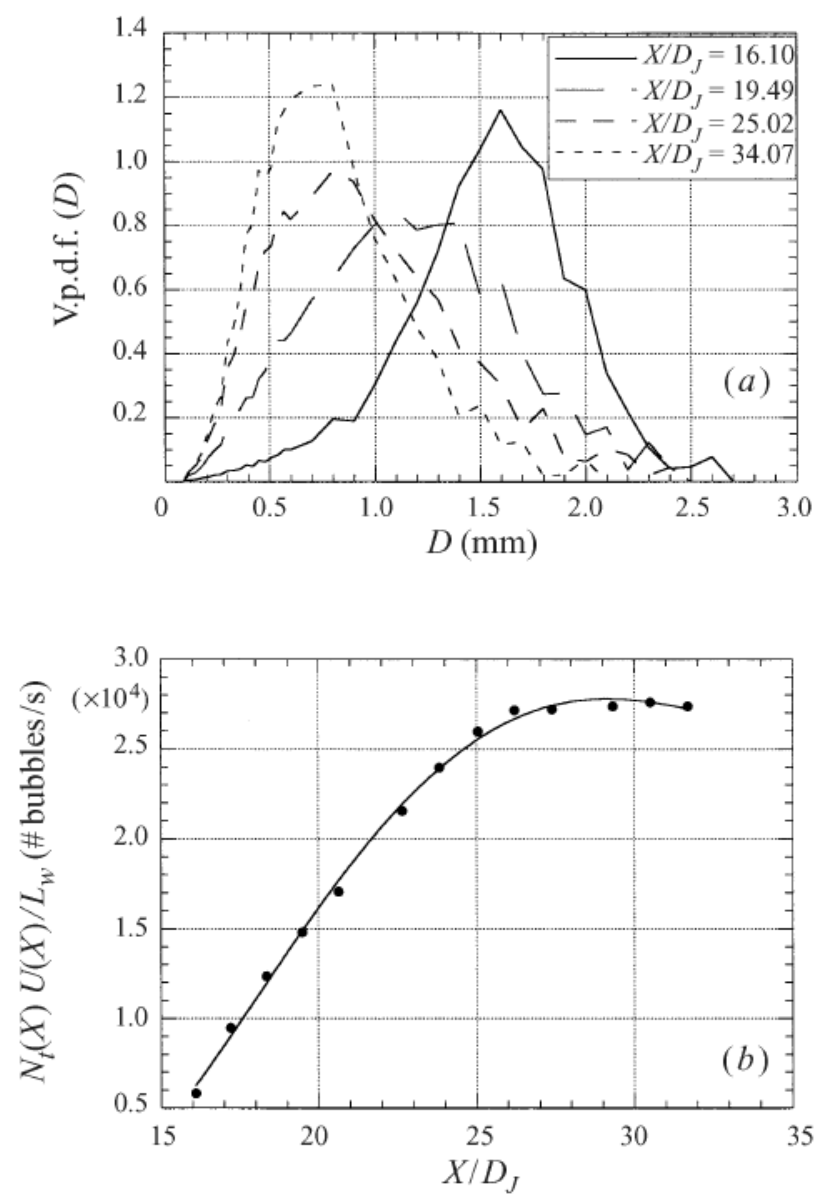

Figure 9. (a) Evolution of the bubble V.p.d.f. (b) Evolution of the flux of bubbles per window. After a frozen V.p.d.f. is achieved, the flux remains at $\approx 2.7 \times 10^{4}$ bubbles $/ \mathrm{s}, D_{a}=0.394 \mathrm{~mm}$, experimental Set $3 \mathrm{a}$.

total number of bubbles measured at the location of the frozen p.d.f. Note that the asymptotic value of the bubbles' flux, $N_{t} U / L_{w}$, reached at the location of the frozen p.d.f. has decreased from $4 \times 10^{4}$ bubbles $/ \mathrm{s}$ in the case of $D_{a_{3}}=1.194 \mathrm{~mm}$ to $2.7 \times 10^{4}$ bubbles $/ \mathrm{s}$ for the case of $D_{a_{1}}=0.394 \mathrm{~mm}$. This decay is a consequence of the apparent increase of the peak in the frozen V.p.d.f., indicating that for the largest injection needle the bubble breakup has produced a larger number of smaller bubbles.

Using the smallest needle $\left(D_{a_{1}}=0.394 \mathrm{~mm}\right)$, we conducted additional experiments by injecting the air at $X / D_{J}=25$, but into a water jet of a much larger Reynolds number, $R_{e}=51000$, corresponding to $U_{o}=17 \mathrm{~m} \mathrm{~s}^{-1}$. These results are shown in figure 11. From the shape of the initial bubble-volume p.d.f., it can be seen that this case resulted in a much broader initial distribution with tails extending up to $5 \mathrm{~mm}$. Qualitatively, the evolution of the V.p.d.f. is also similar to that described above. However, since the values of the dissipation rate are now much smaller, the bubble breakup frequency appears to be much smaller. Thus, even though we begin with much larger bubble sizes, the maximum asymptotic total flux of bubbles measured in this case, $1.05 \times 10^{4}$ bubbles/s, is the smallest of all the cases studied. 

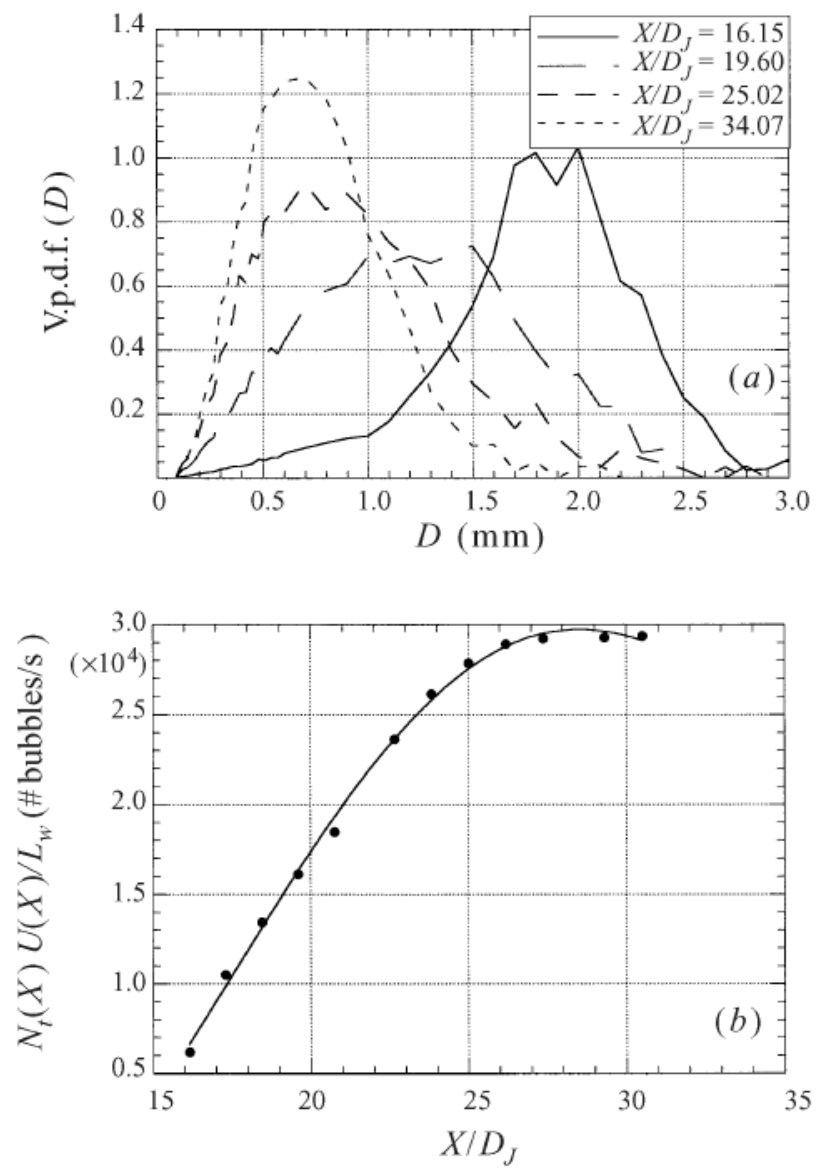

Figure 10. As figure 9 but for $D_{a}=0.584 \mathrm{~mm}$. Experimental Set $3 \mathrm{~b}$. In $(b)$ the flux reaches $\approx 3 \times 10^{4}$ bubbles $/ \mathrm{s}$.

To obtain additional information on the dependence of the bubble breakup frequency on $\epsilon$ and on the bubble size $D$, we conducted a third set of experiments injecting the air at the maximum $\epsilon$ allowed by our experimental techniques, figure 12. This corresponds to the case of $U_{0}=17 \mathrm{~m} \mathrm{~s}^{-1}, R_{e}=51000$, injection point at $X / D_{J}=15$, and $D_{a}=0.394 \mathrm{~mm}$ (Set 2 in table 1 ). As expected from the fact that the dissipation rate of the underlying turbulence is the largest of all our experiments, this case resulted in the maximum asymptotic total flux of bubbles, $\approx 7.4 \times 10^{4}$ bubbles/s, as shown in figure $12(b)$.

3.2. Rate of decay of the number of bubbles of a certain class size

In our experiments, since $\bar{v}(D)=U$, the rate of change of the number of bubbles of a certain bubble-size bin is given by

$$
\frac{\partial(U n)}{\partial x}=\int_{D}^{\infty} m\left(D_{0}\right) f\left(D, D_{0}\right) g\left(D_{0}\right) n\left(D_{0}\right) \mathrm{d} D_{0}-g(D) n .
$$

To measure the breakup frequency we discretized all the measured bubble size p.d.f.s in 10 size bins. If $D_{m}$ is the size representing the largest bin in the distribution, and $n_{m}$ is the number density of bubbles of this maximum size, applying the above 

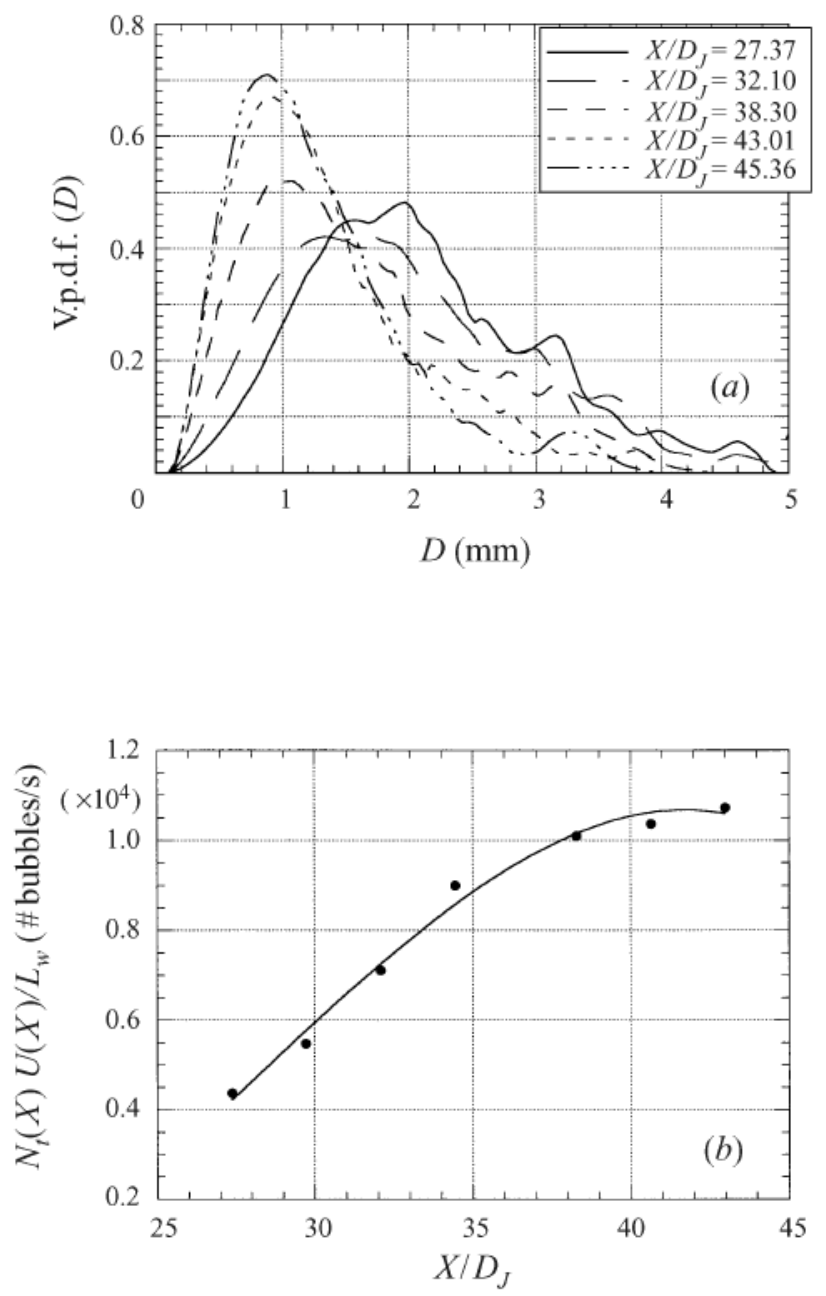

Figure 11. As figure 9 but for $D_{a}=0.394 \mathrm{~mm}$. Experimental Set 1 . In $(b)$ the flux reaches $\approx 1.05 \times 10^{4}$ bubbles $/ \mathrm{s}$.

equation to the class sized $D_{m}$ gives

$$
\frac{\partial\left(U n_{m}\right)}{\partial x}=-g\left(\epsilon, D_{m}\right) n_{m} .
$$

Note that the first term in the right-hand side of equation (3.6) is zero, a consequence of the fact that there are no bubbles larger than $D_{m}$ in the system. Therefore, the breakup frequency can be simply calculated as

$$
g\left(\epsilon, D_{m}\right)=-\frac{1}{n_{m}} \frac{\partial\left(U n_{m}\right)}{\partial x} .
$$

Since, $n_{m}=N_{m} /\left(A L_{w}\right)$, where $N_{m}$ is the number of bubbles of size $D_{m}$ measured in the entire volume of our window of length $L_{w}$ and cross-sectional area $A$ :

$$
g(\epsilon, D)=-\frac{1}{N_{m}} \frac{\partial\left(U N_{m}\right)}{\partial x} .
$$



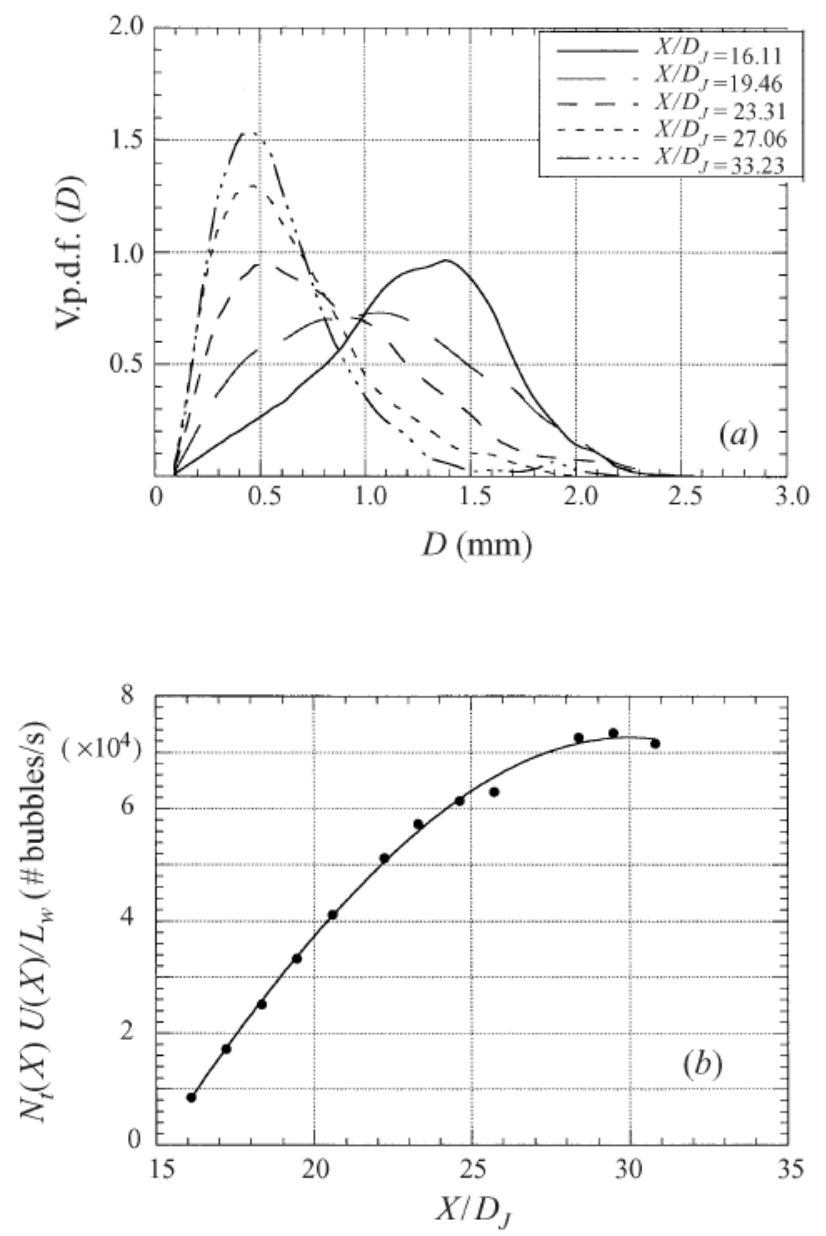

FIGURE 12. As figure 9 but for $D_{a}=0.394 \mathrm{~mm}$. Experimental Set 2 . In $(b)$ the flux reaches $\approx 7.4 \times 10^{4}$ bubbles $/ \mathrm{s}$.

\begin{tabular}{llll}
\hline Case & $D_{m}$ & $D_{m}^{u}$ & $D_{m}^{l}$ \\
Set 1 & 2.75 & 2.95 & 2.55 \\
Set 2 & 1.67 & 1.89 & 1.55 \\
Set 3a & 1.91 & 2.06 & 1.76 \\
$\mathrm{~b}$ & 2.0 & 2.15 & 1.85 \\
$\mathrm{c}$ & 2.08 & 2.23 & 1.93
\end{tabular}

TABLE 2. Mean values of the largest bubble size bin for each experimental Set. $D_{m}^{u}$ is the upper limit and $D_{m}^{l}$ is the lower limit.

For each experimental condition we have different values of $D_{m}$. The values of $D_{m}$ for the five different experiments with the corresponding upper and lower boundaries of the bins are given in table 2. The selection of the bins was done such that in the first measuring window we measured at least $N_{m}=1000$ bubbles over the selected number of measurements performed. The downstream gradient of the flux of bubbles 


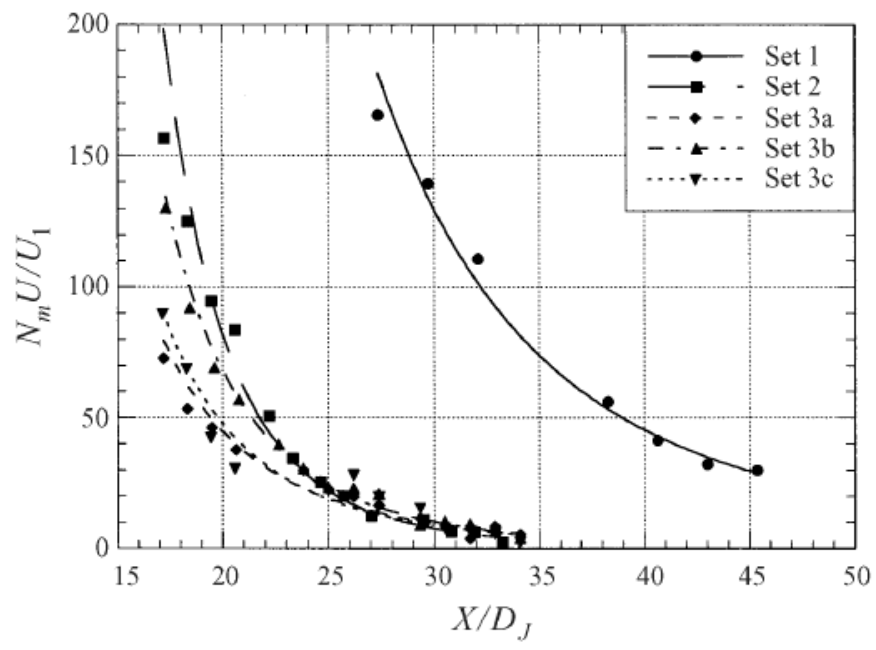

FiguRE 13. Downstream evolution of number of the largest class-size bubbles. The number shown indicates the number of bubbles measured in each position corrected by the ratio of velocities between the measuring window and the first window measured, $N_{c i}=N_{m i} U_{i} / U_{1}$.

of this maximum size, given by equation (3.7) was then measured for each of the five experimental conditions reported.

The measured downstream evolution of $U N_{m}(D)$ corresponding to the largest sizeclass bin, in all the experiments discussed above, is given in figure 13. In each case, the data have been normalized by the velocity at the first measuring location, $U_{1}$. From the measurements of the dissipation rate, $\epsilon$, along the jet's axis, we then calculated the breakup frequency as a function of the dissipation rate, $\epsilon$, and the bubble size, $D$, figure 14. Note that in all cases shown in figure 14, the breakup frequency increases as a power function of the dissipation, with the exponent being approximately constant and equal to 0.3 (from $0.37-0.39$ ). The dependence on $D$ as indicated by the factor in front of the power is not readily apparent, and will be discussed later. In the following section we will discuss a phenomenological model for the bubble's breakup frequency, $g(\epsilon, D)$, and compare it to the above results.

\section{A phenomenological model for the bubble breakup frequency}

Consistent with the experimental evidence, we will assume that the bubbles are injected into a turbulent water flow which is locally homogeneous, isotropic and nearly in equilibrium. Furthermore, the initial size of the bubbles, $D_{0}$, is assumed to be in the inertial subrange, $\eta \ll D_{0} \ll L_{x}$, where $\eta$ is the Kolmogorov microscale of viscous dissipation of the underlying turbulence. Since the one-dimensional energy spectra measured at the bubble injection point show in all cases a $k^{-5 / 3}$ dependence on the wavenumber, $k$, we will also assume that the turbulence is fully developed in the scales of interest, and that local isotropy can be applied as the best approximation to describe the underlying turbulence under which the breakup takes place. We will further assume that the bubble void fraction is always very small $\left(<10^{-5}\right)$, and that there is no two-way coupling between the two phases, i.e. the presence of the air bubbles does not affect the evolution of the turbulence in the water. This assumption is strictly valid for the very small void fraction used in our experiments. However, 

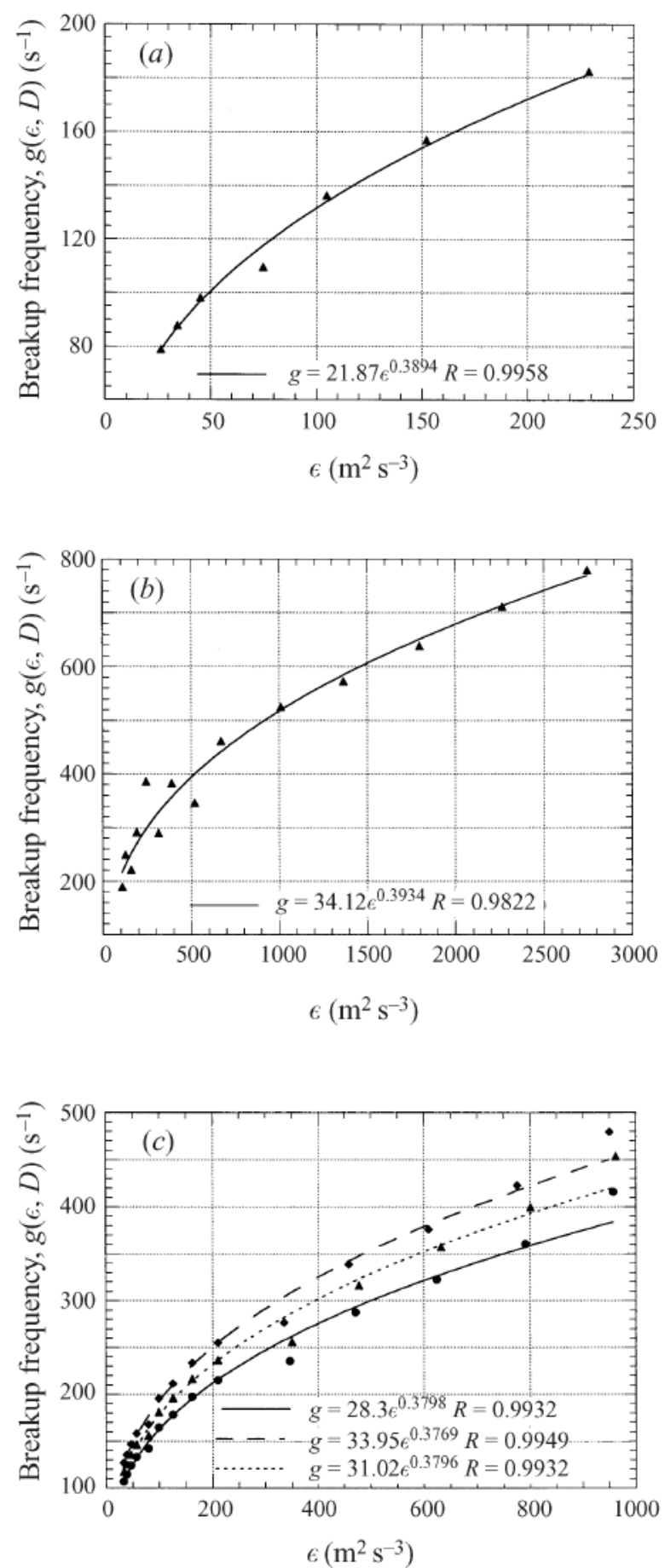

Figure 14. Bubble breakup frequency. (a) Experimental Set 1. (b) Set 2. (c) Set 3.

at large void fractions, the two-way coupling effects may become significant, see Martínez-Bazán (1998).

The basic premise for our model is that for the bubble to break, its surface has to 
deform, and this deformation energy is provided by the turbulent stresses produced by the surrounding water. The minimum energy necessary to deform a bubble of size $D$ is

$$
E_{s}(D)=\pi \sigma D^{2}
$$

Thus, the surface restoring pressure is

$$
\tau_{s}(D)=\frac{6 E_{s}}{\pi D^{3}}=6 \frac{\sigma}{D} .
$$

In the case of an air bubble submerged in a turbulent water flow, the Ohnesorge number is very small, $O h<10^{-2}$ (note that $O h=\mu_{a} / \sqrt{\rho_{a} \sigma D}=2 \times 10^{-2}$ for $D=10 \mu \mathrm{m}$ ), and the internal viscous deformation forces are negligible compared to the surface tension forces. Thus, we will assume that the confinement force is given only by equation (4.2).

One can estimate the average deformation force per unit surface produced by the turbulent stresses resulting from the velocity fluctuations existing in the liquid between two points separated a distance $D$ as

$$
\tau_{t}(D)=\frac{1}{2} \rho \overline{\Delta u^{2}}(D),
$$

where $\rho$ is the density of the water.

When $\tau_{f}(D)>\tau_{s}(D)$, the bubble deforms and eventually breaks up. The equality, $\tau_{f}(D)=\tau_{s}(D)$, defines a critical diameter, $D_{c}$, such that bubbles with $D<D_{c}$ are stable and will never break. A bubble of size $D>D_{c}$ has a surface energy smaller than the deformation energy and, thus, it will break. Following Kolmogorov's universal theory, in the homogeneous and isotropic turbulence conditions of interest here, the mean value of the velocity fluctuations between two points separated a characteristics distance $D$ can be estimated as

$$
\overline{\Delta u^{2}}(D)=\overline{|u(x+D, t)-u(x, t)|^{2}}=\beta(\epsilon D)^{2 / 3},
$$

where, as stated above, $D$ is within the inertial subrange. Equation (4.4) is obtained by integrating over the whole range of turbulent scales, Batchelor (1956).

The critical diameter, $D_{c}=(12 \sigma /(\beta \rho))^{3 / 5} \epsilon^{-2 / 5}$, is then defined by the crossing point of the two curves shown in figure 15 . Note that for a bubble of size $D>D_{c}$, any two points on the surface of the bubble separated a distance $D^{\prime}$ such as $D_{m i n}<D^{\prime}<D$ will experience stresses from the surrounding turbulence with sufficient energy to produce the breakup of the bubble. The value of $D_{\min }$ can be calculated by simply equating the surface energy of a bubble of size $D$ to the deformation energy between points a distance $D_{\min }$ apart:

Thus,

$$
\frac{1}{2} \rho \beta \epsilon^{2 / 3} D_{m i n}^{2 / 3}=6 \frac{\sigma}{D}
$$

$$
D_{m i n}=\left(\frac{12 \sigma}{\beta \rho D}\right)^{3 / 2} \epsilon^{-1} .
$$

The fact that a range of dimensions exists between $D$ and $D_{\min }$ along which the bubble may break, leads to the conclusion that when it breaks, it may result in a wide distribution of daughter bubble sizes. The determination of the p.d.f. of the daughter bubbles is a complex issue which until now has precluded the development of a unified theory (model) to describe the turbulent breakup. However, without addressing here the problem of determining the p.d.f. of the daughter bubbles (presented in a companion paper, Martínez-Bazán, Montañés \& Lasheras 1999), one can determine 


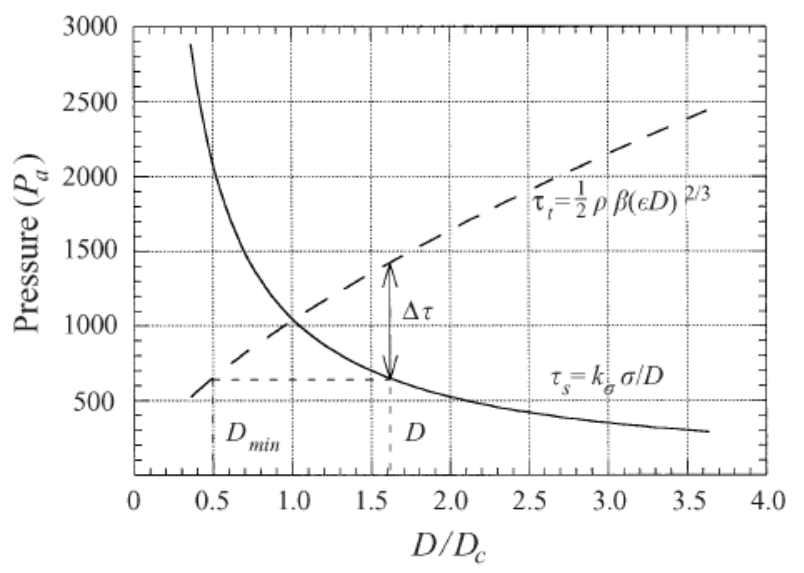

FIGURE 15. Force per unit surface on the bubble. Solid line is the confining force provided by surface tension and the broken line is the one given by the turbulent stresses. The constant $k_{\sigma}=6$ is given in equation (4.2).

the frequency, $g(\epsilon, D)$, at which a bubble of size $D$ will break under turbulence characterized by a dissipation rate, $\epsilon$.

As is the case in any mechanical process, we postulate that the rate at which the breakup process takes place is inversely proportional to the difference between the deformation and confinement forces which produce the deformation of the interface. In other words, we postulate that the larger the difference between the gradient of pressure produced by the turbulent fluctuations on the surface of the bubble $\left(\frac{1}{2} \rho \overline{\Delta u^{2}}(D)\right)$, and the restoring pressures caused by surface tension, $6 \sigma / D$, is, the larger the probability that the bubble will break in a certain time. On the other hand, the breakup frequency should decrease to a zero limit value as this difference of pressures vanishes. Thus, the bubble breakup time can be estimated as

$$
t_{b} \propto \frac{D}{u_{b}}=\frac{D}{\sqrt{\overline{\Delta u^{2}}(D)-12 \sigma /(\rho D)}},
$$

where $u_{b}$ is the characteristic velocity of the bubble breakup process.

The breakup frequency $g(\epsilon, D)$ is then given by

$$
g(\epsilon, D)=\frac{1}{t_{b}}=K_{\mathrm{g}} \frac{\sqrt{\overline{\Delta u^{2}}(D)-12 \sigma /(\rho D)}}{D}=K_{\mathrm{g}} \frac{\sqrt{\beta(\epsilon D)^{2 / 3}-12 \sigma /(\rho D)}}{D},
$$

where the constant $\beta=8.2$ was given by Batchelor (1956), and $K_{g}=0.25$ has been found experimentally. $\dagger$

The dependence of the breakup frequency, given by equation (4.8), on the bubble diameter is shown in figure 16. The breakup frequency is zero for bubbles of size $D \leqslant D_{c}$, and it increases rapidly for bubbles larger than the critical one, $D>D_{c}$. However, it is important to note that after reaching a maximum at $D_{g_{\max }}=1.63 D_{c}$, the breakup frequency decreases monotonically with the bubble size. The maximum

$\uparrow$ The value of $K_{g}=0.25$ has been obtained by best fitting the transient V.p.d.f.s while solving the inverse problem of calculating the daughter p.d.f., see Martínez-Bazán et al. (1999). 


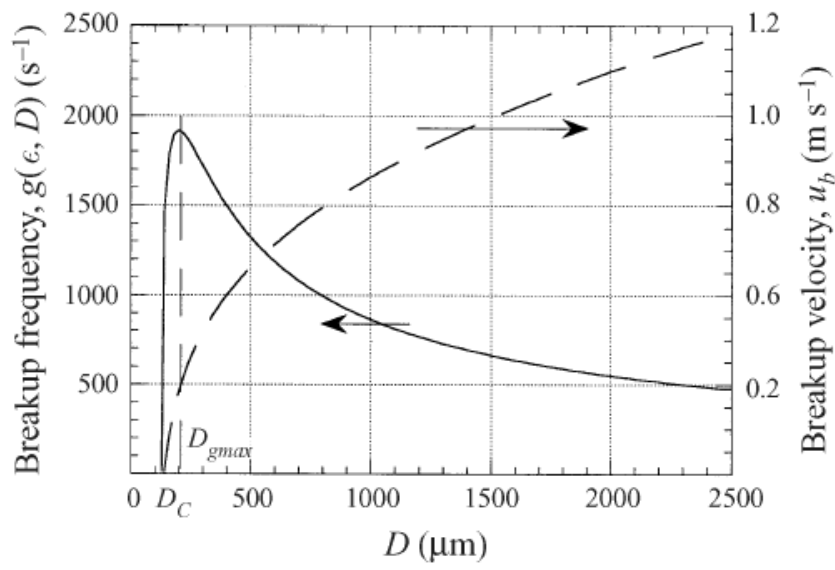

FIgURE 16. Evolution of the bubble breakup frequency and breakup velocity with respect to the diameter of the bubble. $\epsilon=2000 \mathrm{~m}^{2} \mathrm{~s}^{-3}$.

breakup frequency, achieved at $D_{g_{\max }}$, is given by

$$
g_{\max }(\epsilon) \propto(\sigma / \rho)^{-2 / 5} \epsilon^{3 / 5} .
$$

The measured bubble breakup frequency corresponding to the three sets of experiments discussed in $\S 3$ are plotted in figure 17. As discussed in $\S 3$, the breakup frequencies have been calculated from the data corresponding to the largest bubblesize bin which contains enough samples to ensure statistically meaningful results. Due to the discretization needed in our experiments, this bubble-size bin, corresponding to the largest diameters, contains bubbles of sizes between two values, $D_{m}^{u}$ and $D_{m}^{l}$. Thus, in order to test the accuracy of our model given in equation (4.8), we have plotted, jointly with the experimental data, the calculated values of $g\left(\epsilon, D_{m}^{u}\right)$ and $g\left(\epsilon, D_{m}^{l}\right)$ corresponding to the breakup frequencies of the upper, $D_{m}^{u}$, and lower, $D_{m}^{l}$, boundaries of the largest bubble-size bin shown in table 2 .

It should be noted that the model not only describes the qualitative trends, but more importantly, it agrees remarkably well with the measured frequencies. The agreement is within the $10 \%$ maximum experimental error shown by the error bars in figure 17 . Although in our experiments we were only able to vary slightly the bubble's diameter at the point of injection, the experimentally measured dependence of the breakup frequency on the bubble size also appears to be in excellent qualitative agreement with the model. In the experiments shown in figure 17, the characteristic bubble size $D_{0}$ varied from $D_{0}=2.7 \mathrm{~mm}$ in figure $17(a)$ to $D_{0}=1.67 \mathrm{~mm}$ in figure $17(c)$. Notice that, since in all cases $D_{0}>D_{g_{\max }}$, consistent with the model, the breakup frequency decreases as the bubble diameter is increased. Observe that the breakup frequency decreased from $250 \mathrm{~s}^{-1}$ for $D_{0}=2.0 \mathrm{~mm}$ to $200 \mathrm{~s}^{-1}$ for $D_{0}=2.7 \mathrm{~mm}$ when $\epsilon$ is $230 \mathrm{~m}^{2} \mathrm{~s}^{-3}$. Similarly, for $\epsilon=950 \mathrm{~m}^{2} \mathrm{~s}^{-3}$, the breakup frequency decreased from $540 \mathrm{~s}^{-1}$ for $D_{0}=1.67 \mathrm{~mm}$ to $450 \mathrm{~s}^{-1}$ for $D_{0}=2.0 \mathrm{~mm}$.

The dependence of the breakup frequency on the bubble diameter is worthy of further discussion since it appears to have been a source of controversy with previous investigators, see Tsouris \& Tavlarides (1994), Prince \& Blanch (1990) and many others. In the limit of very large bubbles, $D / D_{c} \gg 1$, the surface tension forces become very small and the breakup frequency can be approximated by

$$
g(\epsilon, D) \approx \epsilon^{1 / 3} D^{-2 / 3} .
$$



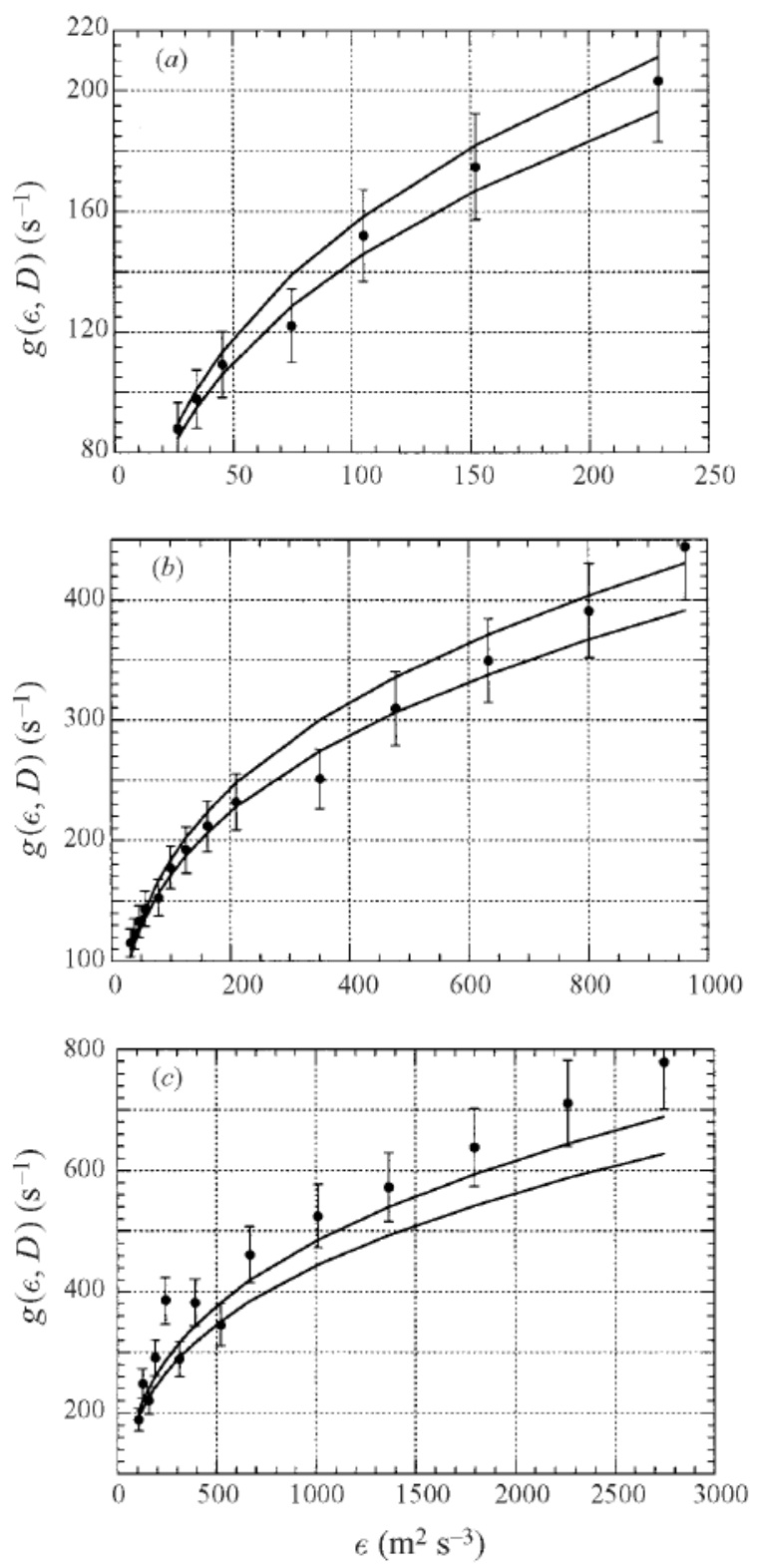

FIGURE 17 . Comparison of experimentally measured bubble breakup frequency with the frequency calculated with model given in equation (4.8). (a) Set $1, D_{0}=2.75 \mathrm{~mm}$. (b) Set $3 \mathrm{~b}, D_{0}=2.0 \mathrm{~mm}$. (c) Set $2, D_{0}=1.67 \mathrm{~mm}$. Error bar indicates an estimated maximum $\pm 10 \%$ experimental error.

This is indeed the dependence that we have measured, since in all our experiments $D / D_{c}$ was always greater than 1.63 . Furthermore, it is important to point out that the diameter for which the breakup frequency is maximum depends only on $D_{c}$,

$$
D_{g_{\max }}=1.63 D_{c}=1.63\left(\frac{12 \sigma}{\beta \rho}\right)^{3 / 5} \epsilon^{-2 / 5},
$$

and that the maximum breakup frequency increases proportionally to $\epsilon^{3 / 5}$ and decays 
with the interfacial tension as $\sigma^{-2 / 5}$,

$$
g_{\max }(\epsilon) \propto\left(\frac{\sigma}{\rho}\right)^{-2 / 5} \epsilon^{3 / 5} .
$$

On the other hand, for small bubbles of sizes smaller than $D_{g_{\max }}$, but comparable to the critical diameter, $D_{c}<D<D_{g_{\max }}$, the turbulent stresses dominate, and the asymptotic dependence of $g(\epsilon, D)$ valid for $D / D_{c} \approx 1$ can be written as

$$
g(\epsilon, D) \propto\left(\frac{\sigma}{\rho}\right)^{-2 / 3} \epsilon^{3 / 5} \sqrt{\frac{D}{D_{c}}-1} .
$$

This is, by the way, the regime in which most of the previous investigations have been conducted. Previous work conducted in turbine mixers generally was done at low $\epsilon$, and in the size range $D_{c}<D<D_{g_{\max }}$. Thus, their reported increase of the breakup frequency with the diameter is also consistent with our present model.

The residence time of the bubbles in our experiments was always shorter than $0.01 \mathrm{~s}$. In the bubble size range for which we have measured the breakup frequency, the time that it takes the bubbles to reach their terminal velocity is about 20 to 30 times larger than their residence time in the breakup region (the time it takes for the bubble p.d.f. to reach a frozen state). Thus, buoyancy effects do not play a role in the breakup process studied here. Similarly, the dynamics of the bubble oscillations also appears to play no role in our bubble breakup. In our experiments, the residence time of the bubbles in the turbulence is shorter than the response time of the bubbles. This assumption is consistent with the measured breakup times which are always one order of magnitude shorter than the characteristic response time of the bubbles to transient excitations.

In summary, the breakup process that we have studied is only controlled by turbulence, and the simplified phenomenological model appears to retain all the important effects since neither buoyancy nor the bubble dynamics should play an important role for the small residence time involved in our experiments. Of course, this effect can be dominant in cases where the residence time of the bubbles in the turbulence is much larger than the response time of the bubble, as has been shown by Risso \& Fabre (1998).

\section{Conclusions}

We have conducted a series of well-controlled experiments where the transient evolution of the bubble size p.d.f. resulting from the breakup of air bubbles of known diameters injected into a fully developed turbulent water flow has been systematically measured using non-intrusive optical techniques. These measurements have been used to calculate the bubble breakup frequency in nearly homogeneous and isotropic turbulent conditions, as a function of their size and of the value of the turbulent kinetic energy of the underlying turbulence.

Experiments performed over a wide range of bubble diameters and turbulent intensities show that the breakup frequency always increases with the value of the dissipation rate of turbulent kinetic energy, $\epsilon$. However, they also show a nonmonotonic dependence of the breakup frequency on the bubble size.

In the past, numerous models have been proposed for the breakage frequency which assumed that the breakup of the drop (or bubble) occurs through the interaction of the drop (or bubble) with an imaginary array of turbulent 'eddies' which are assumed 
to make up the turbulence, see Tsouris \& Tavlarides (1994), Prince \& Blanch (1990), Konno et al. (1983) among others. For example, Tsouris \& Tavlarides (1994) assumed that the drop (or bubble) breakup frequency is equal to the product of a 'drop-eddy' collision rate and a breakage efficiency. These models, which are derived from an extension of the classical kinetic theory of gases (Prince \& Blanch 1990), have the drawback that they require the use of physically questionable closures for the collisions between the particles and the 'eddies', involving assumptions for the eddy-particle collision cross-section, the number of 'eddies' with a certain energy, etc. Furthermore, all these models predict only a monotonic increase of the breakup frequency with both the bubble size and the dissipation rate of turbulent kinetic energy, $\epsilon$.

In this paper we have proposed a phenomenological model for the bubble breakup whose premises are radically different from those used in the past, and do not require invoking the use of an imaginary array of 'eddies' of unknown number and collision cross-section. Our model is based on the premise that the breakup frequency is proportional to the difference between the non-inertial forces which produce the bubble deformation and confinement. This model is shown not only to describe the observed qualitative trends, but also to be in excellent agreement with the measured values. For small bubbles, whose diameters are comparable to the critical diameter, the breakup frequency is shown to increase with the bubble size as $\sqrt{D / D_{c}-1}$, while for large bubbles it decreases as $D^{-2 / 3}$. Furthermore, we have shown the existence of a characteristic bubble size, $D_{g_{\max }}$, for which the breakup frequency is maximum. This maximum frequency increases as $\epsilon^{3 / 5}$ and decreases with the interfacial surface tension as $\sigma^{-2 / 5}$.

Support for this work was provided by a grant from the US Office of Naval Research ONR\# N00014-96-1-0213 (Program officer, Dr Edwin P. Rood). The authors are grateful for the assistance provided to J.L.M. by the Spanish Ministry of Education while he was on leave from the ETS Ingenieros Aeronáuticos, Universidad Politécnica de Madrid (Spain). The material presented here has been extracted from the PhD thesis of the first author (C.M.-B).

This work is dedicated to the memory of Maruja Torralba de Lasheras.

\section{REFERENCES}

Antonia, R. A., Satyaprakash, B. R. \& Hussain, A. K. M. F. 1980 Measurements of dissipation rate and some other characteristics of turbulent plane and circular jets. Phys. Fluids 23, $695-700$.

Baranaey, M. K., Tevenovskiy, Ye. N. \& Tregubova. E. L. 1949 On the measure of minimal fluctuations in a turbulent flow. Dokl. Akad. Navk. SSSR 66, 821-824.

Batchelor, G. K. 1956 The Theory of Homogeneous Turbulence. Cambridge University Press.

Berkman, P. D. \& Calabrese, R. V. 1988 Dispersion of viscous liquids by turbulent flow in a static mixer. $A I C h E$ J. 34, 602-609.

Calabrese, R. V., Chang, T. P. K. \& Dang, P. T. 1986 a Drop breakup in turbulent stirred-tank contactors. Part I. $A I C h E J .32,657-666$.

Calabrese, R. V., WANG, C. Y. \& N. P. BRYner, N. P. $1986 b$ Drop breakup in turbulent stirred-tank contactors. Part III. AIChE J. 32, 677-681.

Coulaloglou, C. A. \& Tavlarides. L. L. 1977 Description of interaction processes in agitated liquid-liquid dispersions. Chem. Engng Sci. 32, 1289-1297.

CutTer, L. A. 1966 Flow and turbulence in stirred tanks. $A I C h E J .12,34$.

Friehe, C. A., VAN AtTa. C. W. \& Gibson, C. H. 1972 Jet turbulence: Dissipation rate measurements and correlations. In Turbulent Shear Flow's, AGARD Conf. Proc, vol. 93, p. 1-18.

Gibson, M. M. 1963 Spectra of turbulence in a round jet. J. Fltid Mech. 15, 161-173. 
HINZE. J. O. 1955 Fundamentals of the hydrodynamics mechanisms of splitting in dispersion process. AIChE J. 1, 289-295.

HiNZE, J. O. 1959 Turbulence, 2nd Edn. McGraw-Hill.

Hussein, H. J., CaPP, S. P. \& George, W. K. 1994 Velocity measurements in a high-Reynolds number, momentum-conserving, axisymmetric, turbulent jet. J. Flifi Mech. 258, 31-75.

Kolmogorov, A. N. 1949 On the breakage of drops in a turbulent flow. Dokl. Akad. Navk. SSSR $66,825-828$.

KoNNO, M., AOKI, M. \& SAITO, S. 1983 Scale-effect on breakup process in liquid-liquid agitated tanks. J. Chem. Engng Japan 16, 312-319.

MartíneZ-BAZÁN, C. 1998 Splitting and dispersion of bubbles by turbulence. PhD thesis, University of California, San Diego.

Martínez-BazÁN, C. Montañés, J. L. \& Lasheras, J. C. 1999 On the breakup of an air bubble injected into a fully developed turbulent flow. Part 2. Size PDF of the resulting daughter bubbles. J. Flaid Mech. 401, 183-207.

Melville, W. K. 1996 The role of surface-wave breaking in air-sea interaction. Ann. Rev. Fluid Mech. 18, 279-321.

Nambiar. D. K. R., Kumar, R., Das, K. S. \& Gandhi. T. R. 1992 A new model for the breakage frequency of drops in turbulent stirred dispersions. Chem. Englig Sci. 47, 2989-3002.

Namblar, D. K. R., Kumar, R. Das, K. S. \& Gandhi, T. R. 1994 A two zone model of breakage frequency of drops in stirred dispersions. Chem. Engng Sci. 49, 2194-2198.

Narsimhan, G., Gupta, J. P. \& Ramkrishna. D. 1979 A model for transitional breakup probability of droplets in agitated lean liquid-liquid dispersions. Chem. Engng Sci. 34, 257-265.

Narsimhan, G., Nejfelt, G. \& Ramkrishna, D. 1984 Breakage functions for droplets in agitated liquid-liquid dispersions. $A I C h E J .30,457-467$.

Prince, M. J. \& BLANCH, H. W. 1990 Bubble coalescence and breakup in air-sparged bubble columns. $A I C h E J .36,1485-1499$,

Risso, F. \& FABRE, J. 1998 Oscillations and breakup of a bubble immersed in a turbulent field. J. Fluid Mech. 372, 323-355.

SATHYAGal, A. N. \& RAmkrishna, D. 1996 Droplets breakage in stirred dispersions. Breakage functions from experimental drop-size distributions. Chem. Engng Sci. 51, 1377-1391.

Sathyagal. A. N.. Ramkrishna, D. \& Narsimhan, G. 1994 Solution of inverse problems in population balances II : particle breakup. Comput. Chem. Engng 19, 437-451.

Tsouris, C. \& TAvlarides, L. L. 1994 Breakage and coalescence models for drops in turbulent dispersions. AIChE J. 40, 395-406.

Wygnanski. I. \& FiedleR. H. K. 1969 Some measurements in the self-preserving jet. J. Finid Mech. 258, $577-612$ 